\title{
On the Numerical Evaluation of Invertible Preconditioners Based on High-Order Discretizations of the Laplace Operator for Linear Systems
}

Chao Lang ( $\sim$ langc14@tsinghua.org.cn )

Beijing Information Science and Technology University

Xiao-Ting Yang

Beijing Information Science and Technology University

\section{Research Article}

Keywords: IIl-posed linear system, Regularization, Laplace operator, Finite difference, Preconditioned iterative methods

Posted Date: October 15th, 2021

DOI: https://doi.org/10.21203/rs.3.rs-959956/v1

License: (c) (i) This work is licensed under a Creative Commons Attribution 4.0 International License.

Read Full License 


\title{
On the numerical evaluation of invertible preconditioners based on high-order discretizations of the Laplace operator for linear systems
}

\author{
Chao Lang ${ }^{[1], *}$ and Xiao-Ting Yang ${ }^{[1]}$, \\ [1] School of Applied Science, Beijing Information Science and Technology University, \\ Beijing 100192, China.
}

Corresponding author: C. Lang (langc14@tsinghua.org.cn)

September 13, 2021

\begin{abstract}
To alleviate the ill-posed condition of linear systems, the discrete Laplace operator is often used as a preconditioner incorporated with iterative methods. However, with a traditional lower-order(typically, second-order) approximation of the Laplace operator it is often difficult to achieve the optimal effect. For this reason, we construct preconditioners based on high-order finite difference discretizations to further develop the potential of the discrete Laplace operator and evaluate their numerical efficiency. The sparse band structure and symmetric property of such high-order preconditioners are derived from the theoretical aspect, revealing the cheap computing cost of the corresponding preconditioning processes for each problem dimension. Numerical experiments are implemented to confirm our analysis and the computing results show advantages of the proposed preconditioned iterative methods compared with the classical methods.
\end{abstract}

Keywords: Ill-posed linear system; Regularization; Laplace operator; Finite difference; Preconditioned iterative methods

\section{Introduction}

In many applications of scientific and engineering computing, such as image deblurring[16, 4], seismic tomography[23] and inverse diffusion[32], the unknown input physical parameters need to be determined from the available output information. They belong to the inverse problem and the corresponding mathematical model can be approximately formulated as the following linear system of equations

$$
A x=b,
$$

*This work was supported by the National Natural Science Foundation (Grant No. 41804051), P.R. China., and the General Science and Technology Project of Beijing Municipal Education Commission (Grant No. KM202111232009). 
where $A \in \mathbb{R}^{m \times n,}, x \in \mathbb{R}^{n}$ and $b \in \mathbb{R}^{m}$ (In general, $m \geq n$ ).

Since the observation error cannot be avoided in practice, the right-hand side vector $b$ is always inaccurate and contaminated by unknown noise term $e$. Typically, it is expressed as

$$
b=\hat{b}+e,
$$

where $\hat{b}$ is the noise-free vector. When the coefficient matrix $A$ is ill-posed which means the singular values decline to zero, the influence of noise on the true solution may be excessively amplified during the computing process of solving (1.1)[9]. The reason can be explained according to the singular value decomposition(SVD)[21]

$$
A=U \Sigma V^{T}
$$

where

$$
U=\left[u_{1}, \ldots, u_{m}\right] \in \mathbb{R}^{m}, V=\left[v_{1}, \ldots, v_{n}\right] \in \mathbb{R}^{n},
$$

have orthogonal columns and $\Sigma \in \mathbb{R}^{m \times n}$ is a diagonal matrix. The first $r$ positive main diagonal elements of $\Sigma: \sigma_{1} \geqslant \sigma_{2} \geqslant \ldots \geqslant \sigma_{r}>0$ are singular values $\left(\sigma_{r} \approx 0\right)$, and $r(\leqslant \min (m, n))$ is the rank of $A$. The least square solution of (1.1) is expressed as

$$
x_{l s}=\sum_{i=1}^{r} \frac{u_{i}^{T} b}{\sigma_{i}} v_{i}=\sum_{i=1}^{r} \frac{u_{i}^{T} \hat{b}}{\sigma_{i}} v_{i}+\sum_{i=1}^{r} \frac{u_{i}^{T} e}{\sigma_{i}} v_{i},
$$

since $\sigma_{r}$ is too small, the noise in the last term on the right side of (1.3) is difficult to control and the accuracy of least square solution $x_{l s}$ is rather low. In this case, the equation (1.1) is called an ill-posed linear system. Although $u_{i}^{T} b$ may satisfy the discrete Picard condition[13], i.e., each $u_{i}^{T} b$ has faster attenuation than $\sigma_{i}$ (e.g. $\left|u_{i}^{T} b\right|=\sigma_{i}^{1+\delta}, \delta>0$ ), this point is not easy to verify in practical computation. Thus, the formula (1.3) often leads to a poor approximation of the true solution for inverse problems and the more efficient method needs to be considered.

The most popular countermeasure is Tikhonov regularization[12], which can be generally expressed as

$$
\min _{x}\left(\|A x-b\|^{2}+\lambda\|P x\|^{2}\right), \lambda>0
$$

here and in the remainder of the paper, $\|\cdot\|$ denotes the Euclidean norm. By choosing the appropriate positive regularization scalar $\lambda$ and smoothing matrix(also called the 'preconditioner' $[6]$ ) $P$, the optimal problem (1.4) can be stably solved to approximate the true solution of the initial linear system (1.1), which can suppress the noise efficiently. When the size of $A$ is small or moderate, the generalized SVD (GSVD) of the regularized coefficient matrix can be performed to directly obtain the solution[3]. However, when considering the large-scale problem, the cost (computing time and memory) of GSVD cannot bear by current machine. Therefore, iterative methods should be considered[1,2], which can make full use of the sparse structure of the coefficient matrix to reduce the computing cost to a reasonable level.

Iterative approaches for solving large-scale linear inverse problem include the Conjugate Gradient Least Square(CGLS)[15], LSQR[28], GMRES[31] and its variant Range-Restricted GMRES (RRGMRES)[18] methods, etc. The CGLS method seeks to solve the normal linear system 
of (1.4), which is symmetric positive definite. During the CGLS iterations, it is not required to explicitly compute $A^{T} A$ to achieve the matrix-by-vector operation $A^{T} A x$. Instead, it only needs to successively compute $y=A x$ and $A^{T} y$. The LSQR method based on the Lanczos bidiagonalization process generates the same Krylov subspace as CGLS to search the optimal solution as that of CGLS[9]. Therefore, these two methods are mathematically equivalent but the LSQR method has better numerical stability. Moreover, neither method constrains the scale parameters of $A$. In other words, regardless of the values of $m$ and $n$, the CGLS and LSQR methods are both suitable and hence have wider applicability. However, during the computing processes of such two methods, the matrix-by-vector operation $A^{T} y$ is inevitable. In some practical situations, the coefficient matrix cannot be expressed explicitly, and it is difficult to determine the transpose matrix $A^{T}$. On the other hand, the GMRES method based on Arnoldi algorithm has good numerical characteristics and the information of $A^{T}$ is not necessary. The RRGMRES method is similar to GMRES and expands the Krylov subspace from the initial vector $A b$ instead of $b$ by Arnold procedure, which can suppress noise to some extent and achieve better numerical performance[18]. The major defects for GMRES class methods include that $A$ must be square and the corresponding approximation subspace for the solution may not be suitable[20], which limit the applicability of such methods.

It is critical to choose the regularization parameter and preconditioner for Tikhonov regularization, which determine the numerical efficiency and the approximate effect of (1.4) on the initial problem (1.1). When the unregularized system is considered, or when Tikhonov regularization is considered with a bad choice (i.e., a too small) regularization parameter, the semiconvergence phenomenon typically happens[8]: at the beginning of iteration, the error of the iterative solution deceases sharply to the minimum at an unknown iterative step and increases in the subsequent iteration. However, the value of parameter may be sensitive and have the adverse effects on the accuracy of iterative solution. There exist some common-used strategies for choosing this scalar, including the discrepancy principle[5], general crossed validation(GCV)[27] and 'L-curve' methods[30]. All of them can avoid semi-convergence phenomenon and provide valid stopping criteria. A further discussion of these strategies can be found in [9].

Effective preconditioners can reduce the ill-posed condition of the linear system (1.1) and make the iterative solution lie in the correct searching subspace which is closer to the true solution[8]. The construction of preconditioners should ensure that the corresponding preconditioning processes incur less computing cost, e.g. small bandwidth, orthogonal project, or circulant structure can be accommodated[19]. Since the coefficient matrix of (1.1) is formulated by discretization of the Laplace operator[11], the preconditioner can also be constructed by this manner. Invertible preconditioners based on second-order discretization of the Laplace operator have been proposed for one-dimensional inverse problems[6]. However, due to rough approximation the discrete Laplace operator is not characterized sufficiently and the numerical performance of such preconditioner strongly depends on the behavior of true solution.

The main purpose of this paper is further evaluating the numerical efficiency of preconditioners based on discrete Laplace operator for linear inverse problems. Concretely, we construct preconditioners based on higher-order(e.g., fourth-order or sixth-order) finite difference numerical stencils to increase the accuracy of discretization. Furthermore, the band structure of the corresponding finite difference based preconditioners are analyzed and we quantitatively derive the dependency between the bandwidth of preconditioners and the order of finite difference discretization to the Laplace operator. Due to the simplicity of the finite difference stencils 
when compared with finite element or other numerical schemes[24], the constructed high-order preconditioners have small bandwidth and the computing cost of the corresponding preconditioning processes can be saved. Then, the numerical experiments of both one-dimensional and two-dimensional inverse problems are performed to investigate our analysis and the obtained results illustrate the superiority of the proposed preconditioners when compared with classical methods in improving computing efficiency.

This paper is organised as follows: In Section 2, we introduce the construction of high-order finite difference based preconditioners and analyze the property of sparse band structure; The preconditioning behavior of the corresponding preconditioned iterative methods is described in Section 3. In Section 4, we perform experiments to examine the numerical characteristics of the constructed preconditioners. Some concluding remarks are listed in Section 5.

\section{High-order finite difference based invertible preconditioners}

Since the expression of the Laplace operator depends on problem scale, the corresponding preconditioners are quite different for each dimension. Firstly, we discuss the preconditioners for one-dimension in detail and use them to construct preconditioners for high-dimensional cases.

The one-dimensional Laplace operator is expressed as one term of the second-order derivative on a single direction. To maintain numerical stability and symmetry, the order of finite difference stencils for discretizing a second-order derivative term should be even[25]. Then, we have

Proposition 2.1. Assuming $h$ denotes the spatial step and $p$ is a positive even scalar, the pth-order finite difference stencils for approximating the second-order derivative of the function $f=f(y)$ at the $i$-th grid node $y_{i}$ can be expressed as

$$
\Delta f=\frac{\partial^{2} f_{i}}{\partial y^{2}}=\frac{1}{h^{2}} \sum_{k=-p / 2}^{p / 2} a_{k} f_{i+k}+\mathcal{O}\left(h^{p}\right),
$$

where $f_{i+k}=f\left(y_{i}+k h\right)$ denotes the function value at the $(i+k)$-th node and $a_{k}$ is the stencil coefficient $(k=-p / 2, \ldots, p / 2)$.

The coefficient $a_{k}(k=-p / 2, \ldots, p / 2)$ can be obtained by expanding the first $p+1$ right-hand terms $f_{i-p / 2}, \ldots, f_{i+p / 2}$ of $(2.1)$ at the $i$-th node $y_{i}$ with Taylor formula. In particular, when considering second-, fourth- and sixth-order cases, the discrete stencils are expressed as

$$
\begin{gathered}
\frac{\partial^{2} f_{i}}{\partial y^{2}}=\frac{1}{h^{2}}\left(f_{i-1}-2 f_{i}+f_{i+1}\right)+\mathcal{O}\left(h^{2}\right), \\
\frac{\partial^{2} f_{i}}{\partial y^{2}}=\frac{1}{h^{2}}\left(-\frac{1}{12} f_{i-2}+\frac{4}{3} f_{i-1}-\frac{5}{2} f_{i}+\frac{4}{3} f_{i+1}-\frac{1}{12} f_{i+2}\right)+\mathcal{O}\left(h^{4}\right),
\end{gathered}
$$

and

$$
\frac{\partial^{2} f_{i}}{\partial y^{2}}=\frac{1}{h^{2}}\left(\frac{1}{90} f_{i-3}-\frac{3}{20} f_{i-2}+\frac{3}{2} f_{i-1}-\frac{49}{18} f_{i}+\frac{3}{2} f_{i+1}-\frac{3}{20} f_{i+2}+\frac{1}{90} f_{i+3}\right)+\mathcal{O}\left(h^{6}\right),
$$


notably, according to this derivation there exists an important property that the stencil coefficients are symmetric with their subscripts

$$
a_{-k}=a_{k}, k=0,1, \ldots, p / 2,
$$

in general, this property is true for any high-order finite difference stencils[33], which implies the symmetry of the corresponding preconditioners since the elements of these matrices are composed of stencil coefficients. We have the following theorem

Theorem 2.1. The high-order invertible preconditioner based on numerical stencil (2.1) for one-dimensional Laplace operator is expressed as

$$
P_{p}^{(1)}=\left(\begin{array}{ccccccc}
a_{0} & \cdots & a_{p / 2} & & & & \\
\vdots & \ddots & & \ddots & & & \\
a_{-p / 2} & \cdots & a_{0} & \cdots & a_{p / 2} & & \\
& \ddots & & \ddots & & \ddots & \\
& & a_{-p / 2} & \cdots & a_{0} & \cdots & a_{p / 2} \\
& & & \ddots & & \ddots & \vdots \\
& & & & a_{-p / 2} & \cdots & a_{0}
\end{array}\right),
$$

moreover, this preconditioner is a symmetric banded matrix and has at most $p+1$ nonzero diagonals.

Proof.

If arranging the function values at grid nodes of the one-dimensional area in a column as

$$
\left(\begin{array}{c}
\vdots \\
f_{i-p / 2} \\
\vdots \\
f_{i} \\
\vdots \\
f_{i+p / 2} \\
\vdots
\end{array}\right)
$$

the right-hand side of (2.1) (neglecting the terms of high-order residuals) is expressed as a product of row vector that contains the stencil coefficients and column vector of function values

$$
\left(\cdots a_{-p / 2}, \cdots, a_{0}, \cdots, a_{p / 2} \cdots\right)\left(\begin{array}{c}
\vdots \\
f_{i-p / 2} \\
\vdots \\
f_{i} \\
\vdots \\
f_{i+p / 2} \\
\vdots
\end{array}\right),
$$


as the index changes, all rows of stencil coefficients with respect to $i$ are collected to formulate the matrix (2.3). It is obvious that $P_{p}^{(1)}$ has $p+1$ nonzero diagonals and that the elements of each diagonal are identical that correspond to one stencil coefficient. Specifically, the value of the main diagonal elements is equal to $a_{0}$. According to $(2.2), P_{p}^{(1)}$ is symmetric.

The matrix expression (2.3) is the general form of preconditioners, which are based on highorder finite difference discretization for one-dimensional Laplace operator. With the rise of $p$, the accuracy of discretization improves, but the bandwidth of preconditioners increases so that more computing cost is needed to invert them.

For two-dimensional case, the Laplace operator is the sum of second-order partial derivatives along two directions. The discrete stencil is similar to that of (2.1), but the grid nodes are located in a two-dimensional square area. The formulation of preconditioners depends strongly on the sorting rules of grid nodes. Thanks to the corresponding discussion in [8], if we sort the grid nodes row by row and moving from left to right for each row, the preconditioners of the two-dimensional case can be formulated by means of Kronecker products and the preconditioners of one-dimensional case.

Proposition 2.2. If considering a square two-dimensional area with the values of the function equal to zero at the boundary, the preconditioners based on the high-order finite difference discretization for two-dimensional Laplace operator are formulated as

$$
P_{p}^{(2)}=P_{p}^{(1)} \otimes I_{n s}+I_{n s} \otimes P_{p}^{(1)}, \quad P_{p}^{(1)}, I_{n s} \in \mathbb{R}^{n s \times n s},
$$

where $I_{n s}$ denotes the identity matrix, $n s=\sqrt{n}$ is the number of grid nodes on one direction, and ' $\otimes$ ' is the notation of Kronecker product.

To evaluate the computing cost during the preconditioning process for preconditioners of the two-dimensional case, the band structure and symmetric property of matrix (2.4) should be analyzed. Analogously, the following theorem can be otained as

Theorem 2.2. The preconditioner $P_{p}^{(2)}$ is a banded matrix for each $p$, and has no more than $2 p+1$ nonzero diagonals. Moreover, $P_{p}^{(2)}$ is symmetric.

Proof.

According to Kronecker product, (2.4) can be rewritten as matrix block form

$$
P_{p}^{(2)}=\left(\begin{array}{ccccccc}
a_{0} I_{n s}+P_{p}^{(1)} & \ldots & a_{p / 2} I_{n s} & & & & \\
\vdots & \ddots & & \ddots & & & \\
a_{-p / 2} I_{n s} & \cdots & a_{0} I_{n s}+P_{p}^{(1)} & \cdots & a_{p / 2} I_{n s} & & \\
& \ddots & & \ddots & & \ddots & \\
& & a_{-p / 2} I_{n s} & \cdots & a_{0} I_{n s}+P_{p}^{(1)} & \ldots & a_{p / 2} I_{n s} \\
& & & \ddots & & \ddots & \vdots \\
& & & & a_{-p / 2} I_{n s} & \cdots & a_{0} I_{n s}+P_{p}^{(1)}
\end{array}\right),
$$

it is easy to find $p$ sub-blocks not located at the main block diagonal and they are all quantity matrices which have only one nonzero diagonal. According to Theorem $2.1, P_{p}^{(1)}$ has no more 
than $p+1$ nonzero diagonals and the main diagonal block of $P_{p}^{(2)}$ has the same number of nonzero diagonals as $P_{p}^{(1)}$. As a result, $P_{p}^{(2)}$ has totally $2 p+1$ nonzero diagonals at most. Owing to the symmetry of $P_{p}^{(1)}$ and $(2.2)$, it is not difficult to verify that $P_{p}^{(2)}$ is symmetric.

Theorem 2.2 reveals that the high-order preconditioners for two-dimensional case still have special band structure, and inverting them in the preconditioning process is also cheap.

Furthermore, analogous to two-dimensional case, the preconditioners based on high-order finite difference stencils for three-dimensional Laplace operator can be derived, and the corresponding property is obtained in a similar way. We just list the corresponding theorem.

Theorem 2.3. (i) When considering a cubic three-dimensional area with zero boundary condition, the preconditioners based on high-order approximation for three-dimensional Laplace operator are formulated as

$$
P_{p}^{(3)}=P_{p}^{(1)} \otimes I_{n s} \otimes I_{n s}+I_{n s} \otimes P_{p}^{(1)} \otimes I_{n s}+I_{n s} \otimes I_{n s} \otimes P_{p}^{(1)},
$$

where $I_{n s}$ is identity matrix and $n s=\sqrt[3]{n}$ is the number of grid nodes on a single direction.

(ii) The preconditioner $P_{p}^{(3)}$ is a symmetric banded matrix, which has no more than $3 p+1$ nonzero diagonals for each pth-order.

To this end, the high-order invertible preconditioners based on discretizing the Laplace operator for each dimension are constructed and the symmetric sparse band structure of these preconditioners are derived. Essentially, it can be attributed to the simple structure of the corresponding finite difference discrete stencils.

\section{Preconditioned iterative method}

When the regularization matrix in (1.4) is square and invertible, the generalized Tikhonov regularization can be equivalently converted to the standard form[9]

$$
\begin{aligned}
& \min _{x}\left(\|A x-b\|^{2}+\lambda\|P x\|^{2}\right) \\
= & \min _{x}\left(\left\|A P^{-1} P x-b\right\|^{2}+\lambda\|P x\|^{2}\right) \\
\triangleq & \min _{z}\left(\left\|A P^{-1} z-b\right\|^{2}+\lambda\|z\|^{2}\right),
\end{aligned}
$$

where $z=P x$ and there exists a bijection between $x$ and $z$ due to the invertibility of $P$. By solving (3.1) with respect to $z$, the iterative solution with $x$ can be obtained correspondingly. At this time, the computing process is affected by the regularized matrix $\tilde{A}=A P^{-1}$.

For broader applicability and better numerical performance, the LSQR method is often employed to solve (3.1). The preconditioned variant of such iterative method is mainly considered in this paper. Actually, the preconditioning behavior of other Krylov subspace iterative methods can be discussed similarly. The LSQR method is based on the Lanczos bidiagonalization procedure[10]. The preconditioned form is listed as

\section{Algorithm 3.1. Lanczos bidiagonalization algorithm (preconditioned version)}

Input: $A, P$ and $b$. 
Set: $\nu_{1}=\|b\|, u_{1}=b / \nu_{1}$.

Set: $v=P^{-T} A^{T} u_{1}, \mu_{1}=\|v\|$, and $v_{1}=v / \mu_{1}$.

for $j=2, \ldots, k+1$

1. Compute $u=A P^{-1} v_{j-1}-\mu_{j-1} u_{j-1}$.

2. Set $\nu_{j}=\|u\|$.

3. Normalize $u_{j}=u / \nu_{j}$.

4. Compute $v=P^{-T} A^{T} u_{j}-\nu_{j} v_{j-1}$.

5. Set $\mu_{j}=\|v\|$.

6. Normalize $u_{j}=u / \mu_{j}$.

end

During this procedure, the preconditioned matrix $\tilde{A}=A P^{-1}$ and its transpose $\tilde{A}^{T}=P^{-T} A^{T}$ need not be computed explicitly, which avoids large amount of computing cost for large-scale problem. Alternatively, achieving $u=A P^{-1} v$ by the following strategy is performed:

(i) Solve the linear system $P w=v$ to obtain $w=P^{-1} v$;

(ii) Do the matrix-by-vector product $u=A w$;

and accomplishing the operation $v=P^{-T} A^{T} u$ by

(i) Do the matrix-by-vector product $w=A^{T} u$;

(ii) Solve the linear system $P^{T} v=w$ to obtain $v=P^{-T} w$.

The two operations listed above are called 'preconditioning process', which is distinguishable from the method without using preconditioner. The computation of $P^{-1}$ and $P^{-T}$ are converted to solve the linear systems with respect to the preconditioner $P$ and its transpose $P^{T}$ and it can be performed by LU factorization. Since the high-order preconditioners introduced in the previous section have symmetric band structure, LU factorization is cheap and the corresponding computing processes are rather stable. The following theorem illustrates this fact

Theorem 3.1. The preconditioner based on the pth-order discretization of two-dimensional Laplace operator is factorized as

$$
P_{p}^{(2)}=L D L^{T}
$$

where $L$ is the unit lower triangular matrix with no more than $p$ nonzero diagonals located at its strictly lower part and $D$ is a diagonal matrix.

Proof.

When performing Gauss elimination to $P_{p}^{(2)}$, the matrix form can be expressed as

$$
P_{p}^{(2)}=L U
$$

where $L$ is a unit strictly lower triangular matrix and $U$ is an upper triangular matrix. During the process of Gauss elimination, the strictly lower triangular part of $L$ keeps consistent with that of $P_{p}^{(2)}$. Since $P_{p}^{(2)}$ is a symmetric banded matrix and has at most $p$ nonzero diagonals located at its strictly lower triangular part, $L$ has no more than $p$ nonzero diagonals at the corresponding location. Moreover, according to the symmetry of $P_{p}^{(2)}$,

$$
\left(P_{p}^{(2)}\right)^{T}=U^{T} L^{T}=P_{p}^{(2)}=L U
$$


by respectively left multiplying $L^{-1}$ and right multiplying $L^{-T}$ to the above equation, it can be obtained

$$
U L^{-T}=L^{-1} U^{T}
$$

note that the left-hand side is an upper triangular matrix and the right-hand is a lower triangular one. Therefore, it must be a diagonal matrix, which is denoted as $D$. As a consequence,

$$
U=D L^{T},
$$

substituting it into (3.3), the expression (3.2) is immediately verified.

In fact, the situations for other dimension are quite similar. Theorem 3.1 explains that the constructed high-order preconditioners and their LU factorization matrices all have sparse band structure, which is especially suitable for large-scale inverse problem. In computing implementation, the LU factorization of the preconditioner is performed before the Lanczos bidiagonalization process of LSQR iteration, and the obtained factorization matrices are stored by sparse storage technique, which can be used to solve the linear systems in the preconditioning process efficiently. In this manner, the factorization of the preconditioner is only performed once during the whole iteration, and both the computing operation and the storage cost for the preconditioning process are magnitude of $\mathcal{O}(n)$. The above analysis illustrates the cheap computing cost of preconditioning process from the theoretical aspect.

Regarding the rest of preconditioned LSQR method, there is little discrepancy with ordinary LSQR method (without a preconditioner). Therefore, we do not repeat it here any more.

\section{Numerical experiments}

In this section, the numerical efficiency of high-order invertible preconditioners is investigated. We use the preconditioners incorporated with the LSQR method (abbreviated as $P_{p}^{(1)}$-LSQR or $P_{p}^{(2)}$-LSQR, $p \geq 4$ ) to solve three typical problems including either one-dimensional or twodimensional case, and compare the computing cost and accuracy with those of classical $P_{2}$-LSQR and ordinary LSQR method without preconditioner. The accuracy of the iterative vector $x_{k}$ with respect to the true solution $x_{*}$ is measured by Relative Error Norm:

$$
R E N=\frac{\left\|x_{k}-x_{*}\right\|}{\left\|x_{*}\right\|}
$$

at the same time, the Relative Residue Norm of the $k$-th step iteration

$$
R R N=\frac{\left\|A x_{k}-b\right\|}{\|b\|}
$$

is also listed, which implies a fact that the initial guess $x_{0}=0$ is chosen in our implementations. The regularization parameter is determined by 'discrepancy principle'[26] with the tolerance constant valuing 1.1 for all experiments. Once the accuracy of $x_{k}$ meets the requirement, the iteration is immediately terminated. At this time the minimum number of iteration(IT) and computing time $(\mathrm{CPU})$ of these methods are respectively recorded and the iterative vector of the corresponding step is taken as the final results of each method. 


\subsection{One-dimensional integral equation problem}

The first example involves the one-dimensional first-kind Fredholm integral equation described by D. L. Phillips[29]

$$
\int_{-6}^{6} K(s, t) x(t)=g(s), \quad s \in[-6,6]
$$

where the kernel function $K(s, t)=\phi(s-t)$, the true solution $x_{*}(t)=\phi(t)$ are defined by the auxiliary function

$$
\phi(t)=\left\{\begin{array}{c}
1+\cos \left(\frac{1}{3} \pi t\right), \quad|t|<3 \\
0 \quad, \quad|t| \geqslant 3
\end{array},\right.
$$

and the right-hand side function

$$
g(s)=(6-|s|)\left(1+\frac{1}{2} \cos \left(\frac{1}{3} \pi s\right)\right)+\frac{9}{2 \pi} \sin \left(\frac{1}{3} \pi|s|\right),
$$

the continuous integral equation (4.1) is discretized by the Galerkin method with an orthonormal box function to derive the linear system (1.1)[14]. After the discretization, the true solution curve and the right-hand vector added by Gaussian noise with the level $\|e\| /\|\hat{b}\|=1 \%$ are as shown in Figure 1, respectively.
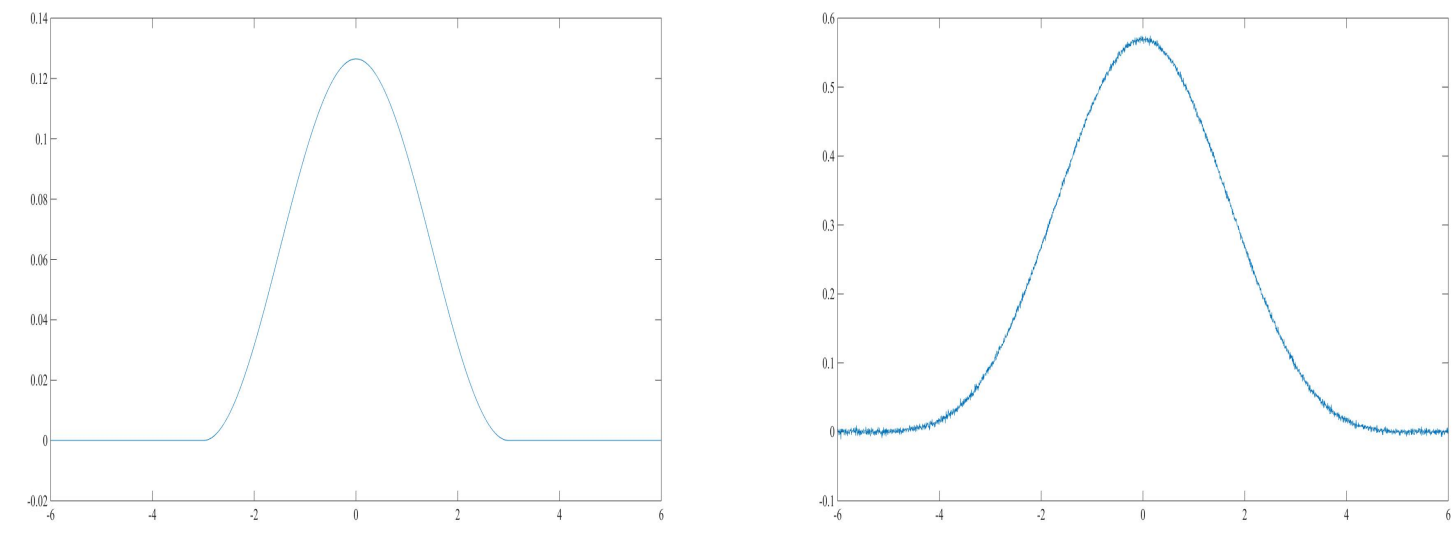

Figure 1: True solution (left) and contaminated right-hand side (right) for Problem 1.

The $P_{16}^{(1)}$-LSQR, $P_{2}^{(1)}$-LSQR and ordinary LSQR methods are employed to solve such an inverse problem, and the obtained solution curves with respect to the true solution are plotted and shown in Figure 2. It is apparent that the solution computed by $P_{16}^{(1)}$-LSQR (dashed-dotted line) conforms better with the true solution (solid line), and there is an obvious difference between the solution of LSQR method (dotted line) and the true solution. Since the solution computed by the $P_{2}^{(1)}$-LSQR method is very close to that of the $P_{16}^{(1)}$-LSQR method, we do not plot the corresponding curve in Figure 2 to avoid confusion. 


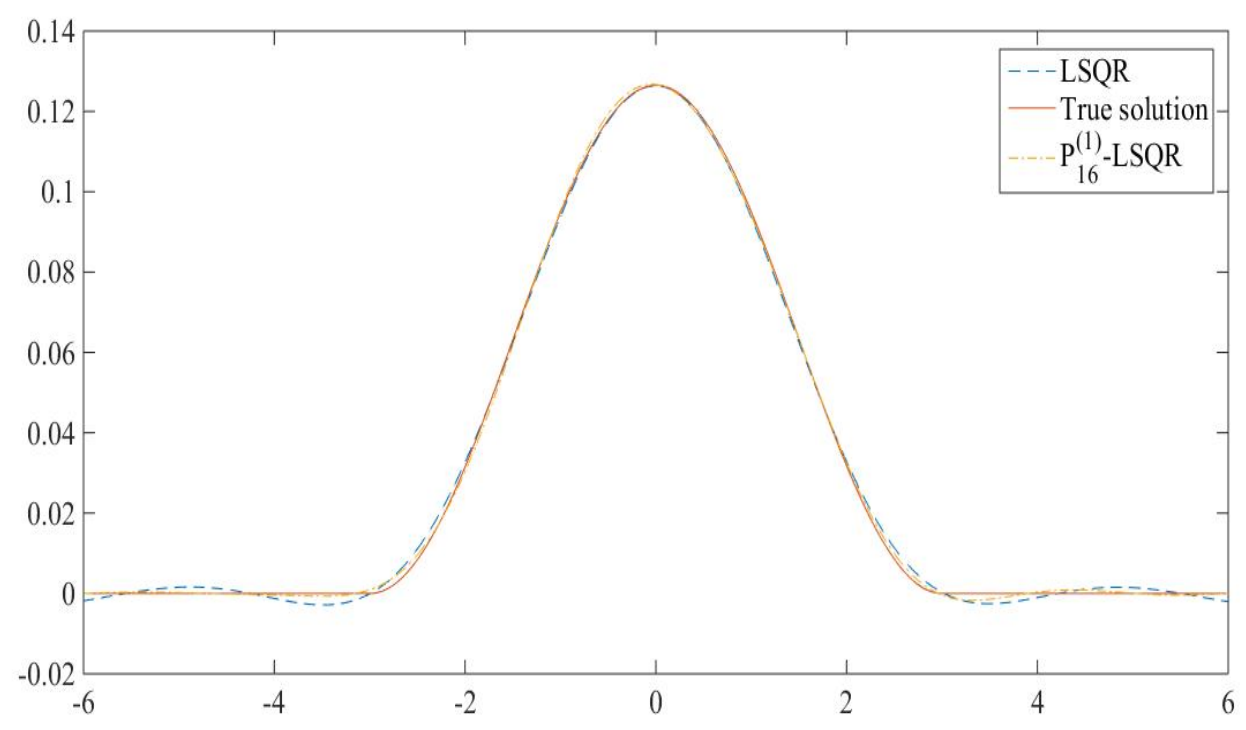

Figure 2: The comparison of solution curves with respect to the true solution for Problem 1.

Table 1: The solution accuracy and computing cost of the three methods for Problem 1.

\begin{tabular}{lllll}
\hline Method & $R E N$ & $R R N$ & IT & CPU(s) \\
\hline$P_{16}^{(1)}$-LSQR & 0.0147 & 0.0101 & 4 & 0.99 \\
$P_{2}^{(1)}$-LSQR & 0.0152 & 0.0101 & 5 & 1.12 \\
LSQR & 0.0264 & 0.0104 & 7 & 1.29 \\
\hline
\end{tabular}

To quantitatively evaluate the accuracy of iterative solutions, the $R E N \mathrm{~s}$ and $R R N \mathrm{~s}$ for these methods are listed in Table 1 . The error norm of $P_{16}^{(1)}$-LSQR is smallest, $3.29 \%$ and $44.3 \%$ less than the errors of the $P_{2}^{(1)}$-LSQR and LSQR methods without preconditioner, respectively. Then, the RRNs of the two preconditioned LSQR methods are both near the noise norm of 0.01, while that of the LSQR method is farthest. It also illustrates the poor performance of the LSQR method to some extent. Moreover, from Table 1 it can be seen $P_{16}^{(1)}$-LSQR method costs least computing time since it needs fewest iterative steps to reach the minimum error norm.

In addition, we further investigate the numerical efficiency of constructed preconditioners with resepct to the discrete order of the Laplace operator by this numerical example. We use the $P_{p}^{(1)}$-LSQR $(p=2,4, \ldots, 16)$ methods to solve this problem and plot the corresponding $R E N$ curve with respect to the order $p$, as shown in Figure 3. The error norm decreases with the order number, and $P_{16}^{(1)}$ provides the best numerical performance. Thus, in practical computation, we can choose the relatively higher-order preconditioned iterative method as possible according to available computing resources. 


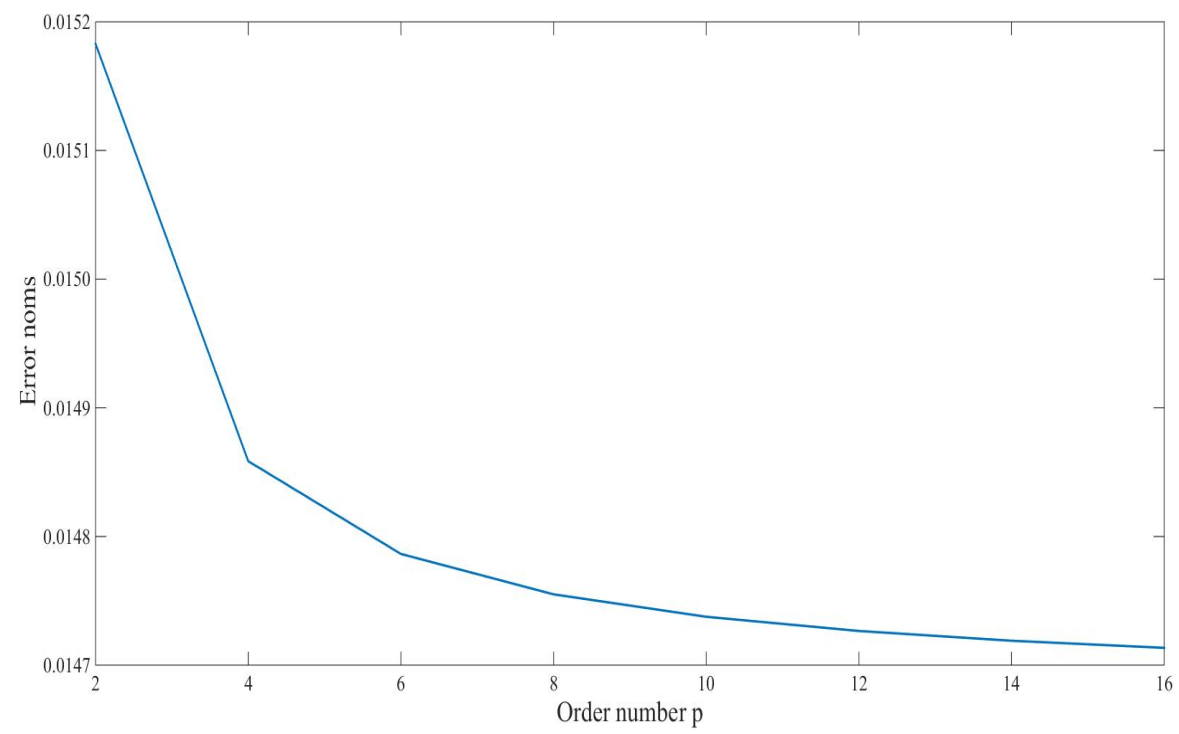

Figure 3: The error norms versus order number of the numerical stencils for Problem 1.

\subsection{Two-dimensional image deblurring problem}

The image deblurring problem is one of the current research hotspots that involves restoring the image which is blurred seriously and contaminated with noise. Its mathematical essence is an inverse problem that can be expressed as the two-dimensional integral equation

$$
g(s)=\int k(s, t) x(t) d t, \quad s, t \in \mathbb{R}^{2},
$$

where the kernel function $k(s, t)$ is also called the 'point spread function', which determines the manner of blurring from the initial image. In this subsection, this function is used to simulate a rotational blurring motion around the center of the image[17].

The discretization of (4.2) is the same as that of [8]. Moreover, the true model and righthand vector $\hat{b}$ with $1 \%$ Gauss noise added are shown in Figure 4, respectively. This figure shows two gray-scale images drawn by the gray-scale value of $256 \times 256$ pixel points located in a two-dimensional square area.

We use the $P_{6}^{(2)}$-LSQR method and compare the computing results with those of the $P_{2}^{(2)}$ LSQR and ordinary LSQR method as well. The image solutions of these methods are shown in Figure 5. The iterative results computed by the $P_{6}^{(2)}$-LSQR method is closest to the true solution, and the corresponding image resolution is the highest among three images. The $P_{2}^{(2)}-$ LSQR performs better than the LSQR method, but there are still some slight blurring and traces of rotation at the center of image. For the image corresponding to the LSQR method, there are obvious errors on the background, and the resolution is not high.

In addition, the $R E N \mathrm{~s}, R R N \mathrm{~s}$, iterative number and computing time are listed in Table 2 to compare the efficiency of the corresponding methods. We find that both the $R E N$ and computing time of the $P_{6}^{(2)}$-LSQR method are smallest, and the solution accuracy is increased 

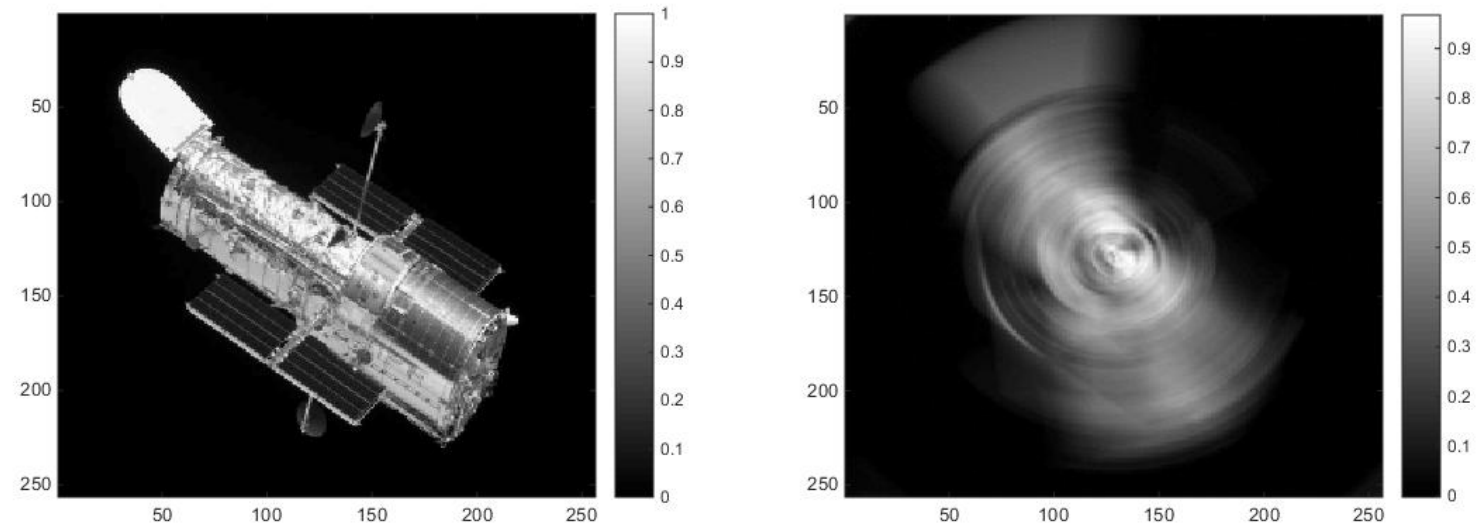

Figure 4: True solution (left) and contaminated right-hand side (right) for Problem 2.

approximately by $10.9 \%$ of $P_{2}^{(2)}$-LSQR and $29.1 \%$ of LSQR methods, respectively. When observing $R R N \mathrm{~s}$, it can be seen they are all around 0.01 and the $R R N \mathrm{~s}$ of the preconditioned LSQR methods are also closer than that of the ordinary LSQR method to this noise level.

Table 2: The solution accuracy and computing cost of the three methods for Problem 2.

\begin{tabular}{lllll}
\hline Method & $R E N$ & $R R N$ & IT & CPU(s) \\
\hline$P_{6}^{(2)}$-LSQR & 0.1499 & 0.0103 & 46 & 13.99 \\
$P_{2}^{(2)}$-LSQR & 0.1683 & 0.0104 & 49 & 14.68 \\
LSQR & 0.2113 & 0.0109 & 53 & 16.71 \\
\hline
\end{tabular}

\subsection{Two-dimensional seismic tomography problem}

As an important research field in geophysics, seismic tomography is also an inverse problem from the view point of computing mathematics[22]. Its primary task is to take use of the seismic observation data at the surface to compute subsurface physical parameters, which is critical to explore the deep structure of the Earth[24].

In this subsection, we use the MATLAB package 'IR Tools'[8] to generate the linear system (1.1). To better address practical applications, the model 'tectonic' in 'IR Tools' undergoes some changes, especially when an anomaly block is added at the lower-left part of the computing area (shown on the left side of Figure 6). The observation data arranged in the right-hand vector of (1.1) is also contaminated by $1 \%$ Gauss noise, as shown on the right side of Figure 6 . It is worth mentioning that the coefficient matrix $A$ in (1.1) is no longer square. In detail, the matrix scale parameters $m=512, n=256$ are set in this case, and the size of the two-dimensional computing area is $256 \times 256$. 

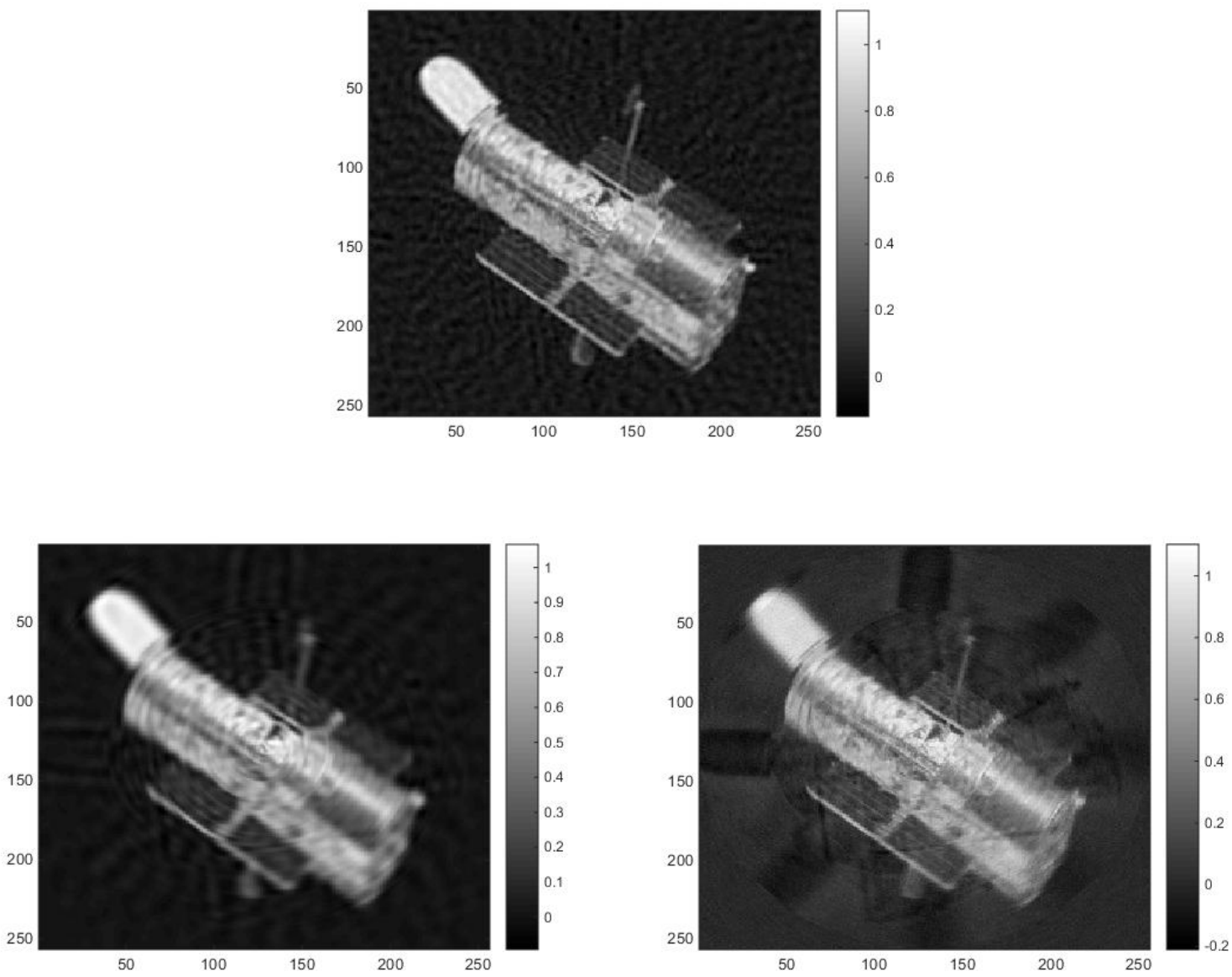

Figure 5: Image solutions computed by $P_{6}^{(2)}-\operatorname{LSQR}\left(\right.$ top), $P_{2}^{(2)}-\operatorname{LSQR}($ left bottom), and LSQR(right bottom) methods for Problem 2.

The $P_{4}^{(2)}$-LSQR method is employed for this problem and compared with the $P_{2}^{(2)}$-LSQR and ordinary LSQR methods. The iterative solutions of the three methods are shown in Figure 7. Similarly, the solution computed by the $P_{4}^{(2)}$-LSQR method is closest to the true solution, and the corresponding image has the highest resolution. For the images obtained by the other two methods, the backgrounds are obviously confused. Besides, the numerical performance of the $P_{2}^{(2)}$-LSQR method is superior to that of the non-preconditioned LSQR method. According to this numerical example, the high-order based preconditioners are still efficient when the shape of the coefficient matrix is not square (to be specific, $m>n$ ).

Accordingly, the $R E N \mathrm{~s}$ and $R R N$ s of the three methods are listed in Table 3. The $R E N$ with respect to the $P_{4}^{(2)}$-LSQR method is also the smallest and decreases by $7.58 \%$ of the $P_{2}^{(2)}$-LSQR and $37.6 \%$ of the LSQR method. Moreover, the RRNs of the preconditioned LSQR methods are closer to the noise level than that of the ordinary LSQR method. Similar to the previous two experiments, $P_{4}^{(2)}$-LSQR method needs the least iterative numbers and computing time when compared with the other two methods to reach the minimum error norm. 

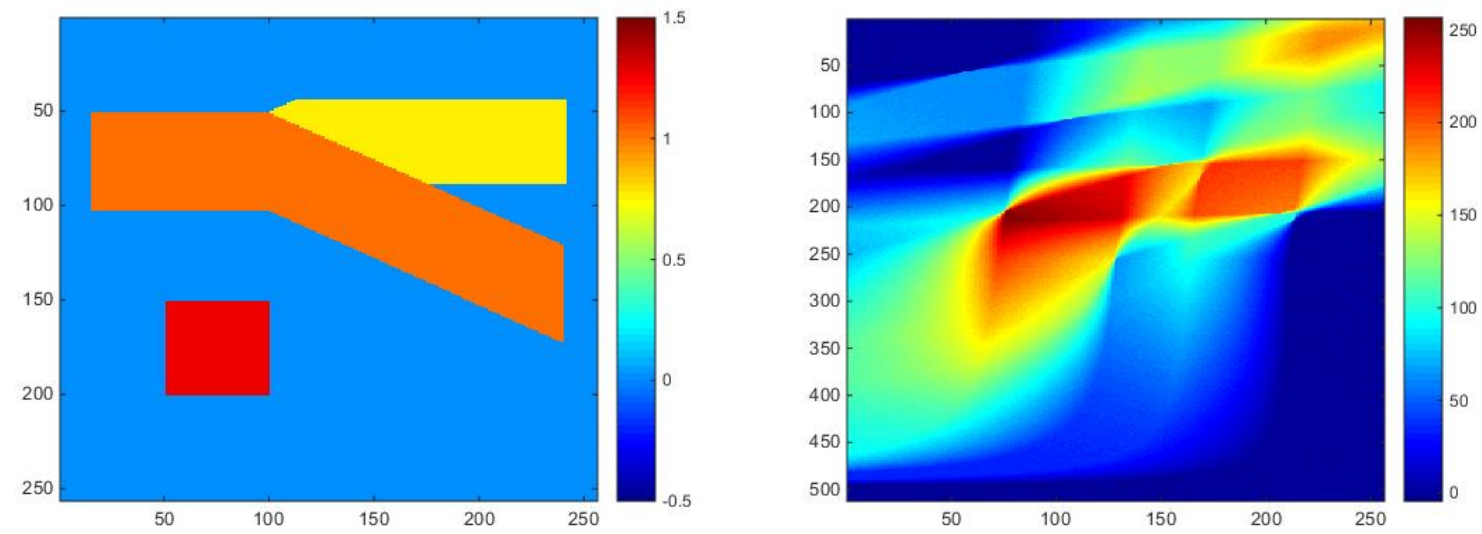

Figure 6: True solution (left) and contaminated observation data (right) for Problem 3.

Table 3: The solution accuracy and computing cost by the three methods for Problem 3.

\begin{tabular}{lllll}
\hline Method & $R E N$ & $R R N$ & IT & CPU(s) \\
\hline$P_{4}^{(2)}$-LSQR & 0.2220 & 0.0113 & 81 & 41.65 \\
$P_{2}^{(2)}$-LSQR & 0.2402 & 0.0113 & 87 & 43.72 \\
LSQR & 0.3560 & 0.0116 & 94 & 48.69 \\
\hline
\end{tabular}

\section{Concluding remarks}

In this paper, we construct invertible preconditioners by high-order finite difference discretization of the Laplace operator incorporated with Krylov subspace iterative method (specifically, LSQR) to further increase the numerical effects for ill-posed linear systems. The symmetric sparse band structure of the preconditioner is derived to illustrate the cheap computing cost including memory and computing operation of the corresponding preconditioning processes from the theoretical aspect (to be specific, both of them reach the magnitude of $\mathcal{O}(n)$ ). In fact, it benefits from the simplicity of finite difference stencils, and is especially suitable for large-scale inverse problems. To confirm our analysis, numerical experiments including one-dimensional and two-dimensional typical inverse problems are performed to evaluate the efficiency of the corresponding preconditioned LSQR method when compared with classical $P_{2}$-LSQR and ordinary LSQR method without preconditioners. The numerical results show that the high-order preconditioned LSQR method costs least iterations and computing time in all cases and the corresponding solutions are closest to the true solutions, which approximately increase the accuracy by at most $10.9 \%$ over the $P_{2}$-LSQR method and $44.3 \%$ over the LSQR method, respectively. In addition, from the final seismic tomography experiment it can be seen when the coefficient matrix of the linear inverse problem is not square (e.g. more rows than columns), the superiority of the proposed preconditioners in improving efficiency over the classical methods is still 

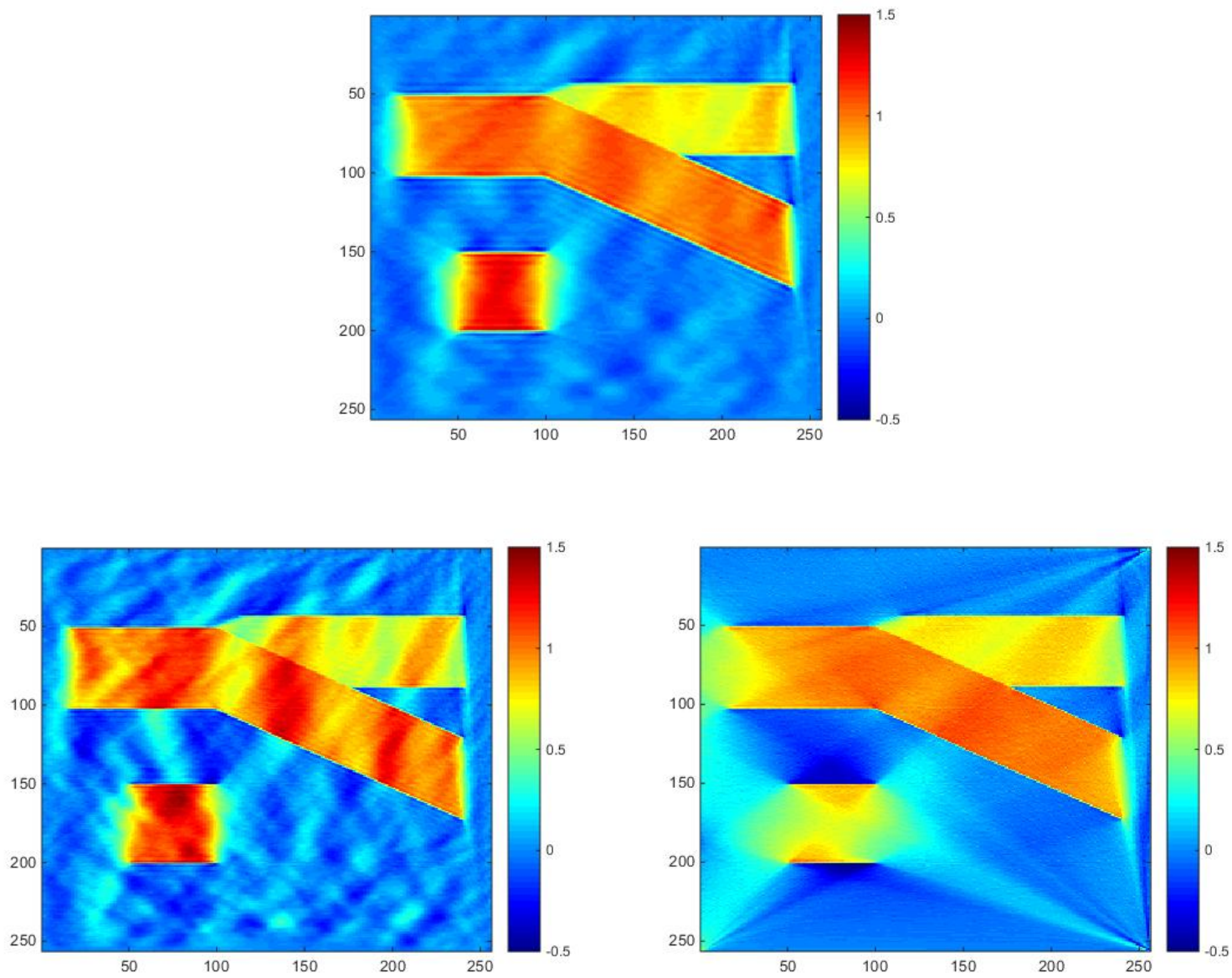

Figure 7: Image solutions computed by $P_{4}^{(2)}$-LSQR(top), $P_{2}^{(2)}$-LSQR(left bottom), and LSQR(right bottom) methods for Problem 3.

apparent, which shows the wide applicability of such preconditioners.

To this end, it is necessary to claim that constructing efficient methods for ill-posed linear systems is not easy. In the future, we plan to further study efficient preconditioners by sufficiently taking use of prior information [7] and the property of the coefficient matrix for certain problems.

\section{References}

[1] Bai, Z.-Z. and Wu, W.-T. (2018). On relaxed greedy randomized Kaczmarz methods for solving large sparse linear systems. Applied Mathematics Letters, pp: 21-26.

[2] Bai, Z.-Z. and Wu, W.-T. (2019). On greedy randomized coordinate descent methods for solving large linear least-squares problems. Numerical Linear Algebra With Applications, volume 26(4). 
[3] Bai, Z.-Z. and Wu, W.-T. (2019) On partially randomized extended Kaczmarz method for solving large sparse overdetermined inconsistent linear systems. Linear Algebra and its Applications, pp: 225-250.

[4] Buccini, A. and Donatelli, M. (2020) A multigrid frame based method for image deblurring. Electronic Transactions on Numerical Analysis, volume 53, pp: 283-312.

[5] Calvetti, D., Reichel, L., and Shuibi, A. (2005) Tikhonov regularization of large symmetric problems. Numerical Linear Algebra with Applications, volume 12(2-3), pp: 127-139.

[6] Calvetti, D., Reichel, L., and Shuibi, A. (2005) Invertible smoothing preconditioners for linear discrete ill-posed problems. Applied Numerical Mathematics, volume 54(2), pp: 135-149,

[7] Calvetti, D., and Somersalo, E. (2005) Priorconditioners for linear systems. Inverse problems, volume 21(4), pp: 1397.

[8] Gazzola, S., Hansen, P.C., and Nagy, J.G. (2019) IR Tools: a MATLAB package of iterative regularization methods and large-scale test problems. Numerical Algorithm, volume 81, pp: 773-811.

[9] Gazzola, S., Novati, P., and Russo, M.R. (2015) On Krylov projection methods and Tikhonov regularization. Electronic Transactions on Numerical Analysis, volume 44(1), pp: 83-123.

[10] Golub, G. and Kahan, W. (1965) Calculating the singular values and pseudo-inverse of a matrix. Journal of the Society for Industrial and Applied Mathematics Series B Numerical Analysis, volume 2, pp: 205-224.

[11] Hanke, M. (1992) Regularization with Differential Operators: An Iterative Approach. Numerical Functional Analysis and Optimization, volume 13(5-6), pp: 523-540

[12] Hanke, M. and Hansen, P.C. (1993) Regularization methods for large-scale problems. Surveys on Mathematics for Industry, volume 3, pp: 253-315.

[13] Hansen P.C. (1990) The discrete Picard condition for discrete ill-posed problems. BIT Numerical Mathematics, volume 30, pp: 658-672.

[14] Hansen, P.C. (1994) REGULARIZATION TOOLS: A Matlab package for analysis and solution of discrete ill-posed problems. Numerical Algorithm, volume 6, pp: 1-35.

[15] Hansen, P.C. (2010) Discrete inverse problems: insight and algorithms. SIAM Philadelphia.

[16] Hansen, P.C., Nagy, J.G., and O‘Leary, D.P. (2006) Deblurring Images: Matrices, Spectra and Filtering. SIAM, Philadelphia PA.

[17] Hansen, P.C., Nagy, J.G., and Tigkos, K. (2014) Rotational image deblurring with sparse matrices. BIT Numerical Mathematics, volume 54, pp: 649-671.

[18] Hochstenbach, M.E., Mcninch, N., and Reichel, L. (2012) Discrete ill-posed least-squares problems with a solution norm constraint. Linear Algebra and its Applications, volume 436, pp: 3801-3818. 
[19] Hochstenbach, M.E. and Reichel, L. (2010) An iterative method for Tikhonov regularization with a general linear regularization operator. Journal of Integral Equations Applications, volume $22(3)$, pp: 465-482.

[20] Jensen, T.K. and Hansen, P.C. (2007) Iterative regularization with minimum-residual methods. BIT Numerical Mathematics, volume 47(1), pp: 103-120.

[21] Kilmer, M.E., Hansen, P.C., and Espanol, M.I. (2007). A projection-based approach to general-form Tikhonov regularization. SIAM Journal on Scientific Computing, volume 29(1), pp: $315-330$.

[22] Liu, S.-L., Suardi, I., and Zheng, M. (2019) Slab morphology beneath northern Sumatra revealed by regional and teleseismic traveltime tomography. Journal of Geophysical Research, volume 124(10), pp: 10544-10564.

[23] Liu, S.-L., Suardi, I., Yang, D.-H., Wei, S.-J., and Tong, P. (2018) Teleseismic traveltime tomography of northern Sumatra. Geophysical Research Letters, volume 45(24), pp: 231-239.

[24] Liu, S.-L., Yang, D.-H., Dong, X.-P., and Qian, C. (2017) Element-by-element parallel spectral-element methods for 3-D teleseismic wave modeling. Solid Earth, volume 8(5), pp: 969-986.

[25] Liu, S.-L., Yang, D.-H., and Ma, J. (2017) A modified symplectic PRK scheme for seismic wave modeling. Computers \& Geosciencs, volume 99(2), pp: 28-36.

[26] Morigi, S., Reichel, L., and Sgallari, F. (2006). An iterative Lavrentiev regularization method. BIT Numerical Mathematics, volmue 46(3), pp: 589-606.

[27] Novati, P., and Russo, M.R. (2014) A GCV based Arnoldi-Tikhonov regularization method. Bit Numerical Mathematics, volume 54, pp: 501-521.

[28] Paige, C.C. and Saunders, M. A. (1982) LSQR: an algorithm for sparse linear equations and and sparse least squares. ACM Transactions on Mathematical Software, volume 8, pp: 43-71.

[29] Phillips, D. L. (1962) A technique for the numerical solution of certain integral equations of the first kind. Journal of the ACM, volume 9, pp: 84-97.

[30] Rodruguez, G. and Theis, D. (2005) An algorithm for estimating the optimal regularization parameter by the L-curve, Rendiconti Lincei-Mathematica E Applizioni, volume 25(7), pp: 69-84.

[31] Saad, Y. and Schultz, M. H. (1986) GMRES: a generalized minimal residual method for solving nonsymmetric linear systems. SIAM Journal on Scientific and Statistical Computing, volume 7, pp: 856-869.

[32] Tao, M., Geng, B., and Ren, J. (2013) Inverse estimation of the initial condition for the heat equation. International Journal of Pure and Applied Mathematics, volume 82, pp: 581-593.

[33] Yang, D.-H., Teng, J.-W., Zhang, Z.-J., and Liu, E.-R. (2003) A nearly analytic discrete method for acoustic and elastic wave equations in anisotropic media. Bulletin of the Seismological Society of America, volume 93(2), pp: 882-890. 